\title{
Use of 99mTc-labelled Anti-TNF Monoclonal Antibodies to Assess Patients Affected by Inflammatory Arthropathies
}

\section{Fulvia Ceccarelli ${ }^{1}$, Carlo Perricone ${ }^{1}$, Filippo Galli $^{2}$, Guido Valesini ${ }^{1}$ and Fabrizio Conti ${ }^{{ }^{\star}}$}

${ }^{1}$ Rheumatology, Dipartimento di Medicina Interna e Specialità Mediche, Sapienza Università di Roma, Viale del Policlinico 155, 00161, Roma, Italy

${ }^{2}$ Nuclear Medicine Unit,Faculty of Medicine and Psychology,Department of Medical-Surgical Sciences and Translational Medicine, "Sapienza" University, Ospedale S. Andrea, Via di Grottarossa 1035; 00189 Rome, Italy

\section{Abstract}

Nuclear medicine techniques have been applied for many years to assess the activity in patients with different inflammatory arthropathies. The recent development of specific radiopharmaceuticals suggested the possibility to use nuclear medicine in order to clarify the disease mechanisms and predict response to treatment. In this review, we aimed at presenting data concerning the use of ${ }^{99 \mathrm{~m}}$ Tc-labelled anti-TNF monoclonal antibodies. Few studies have been conducted in patients affected by Rheumatoid Arthritis and Spondyloarthropathies, treated for systemic or mono-articular involvement, showing the possible use of this specific scintigraphy.

\section{Publication History:}

Received: March 20, 2015

Accepted: April 12, 2015

Published: April 14, 2015

\section{Keywords:}

Adalimumab, Biomarkers, Diagnosis, Infliximab, Response.

\section{Introduction}

Nuclear medicine techniqueshave been applied for many years to assess the activity status in patients affected by inflammatory arthropathies. Recently, the development of specific radiopharmaceuticals suggested the possibility to use nuclear medicine techniques not only in the assessment and monitoring of patients, but also to clarify the disease mechanisms. Moreover, these new imaging modalities could help the physician for therapy decision-making and follow-up[1]. In particular, recentstudies

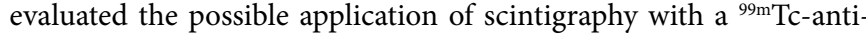
tumor necrosis factor (TNF)monoclonal antibody (mAb)in the assessment of patients affected by inflammatory bowel diseases and inflammatory arthropathies, such as rheumatoid arthritis (RA) [2-3] Biological agents directed against TNF revolutionized the therapeutic approach of these patients, due to the innovative mechanism of action, based on the ability to recognize specific molecular or cellular targets involved in the disease pathogenesis. The administration of anti-TNF drugsdetermined a dramatic improvement in the prognosis of patients refractory or intolerant to conventionaldisease modifying anti-rheumatic drugs(DMARDs) treatment. Randomized controlled trials demonstrated the efficacy of these drugs to prevent the development of bone erosive damage, with consequent better profile in terms of remission status and prevention of long-term disability [4]. The identification of pivotal role of pro-inflammatory cytokines in the pathogenesis of inflammatory diseases determined the attempt to develop drugs specifically targeting the molecules involved in the disease processes. Indeed, TNF is a pro-inflammatory cytokine able to promote synovial inflammation and bone and cartilage erosions [5]. Produced byseveral different immune and non-immune cell types, it was recognized as a key factor in the inflammatory processof arthropathies, such as RA, psoriatic arthritis (PsA) and ankylosing spondylitis (AS) [6]. To date, five different anti-TNF drugshave been developed: infliximab, adalimumab, golimumab, certolizumabpegol, which are mAbs or fragments thereof, and etanercept, a genetically engineered fusion protein composed of a dimer of the extracellular portions of human TNFR2 fused to the Fc portion of a human IgG1 [6]. Despite therecognized efficacy of anti-TNF agents, approximately one third of patients have to discontinue the treatment due to inefficacy or intolerance. In these cases, the switching to another anti-TNF agent could represent a valid option due to significant differences in terms of molecular structure, pharmacokinetics, interactions with TNF, generation of antibodies, induction of apoptosis, and dosing regimen among the TNF antagonists [4]. More recently, other molecular or cellular targets involved in the pathogenesis of the autoimmune diseaseshave been identified, such asthepro-inflammatory cytokines interleukin-1 and interleukin-6, CTLA-4, and molecules involved in the activation, differentiation and maturation of $B$ cells. These drugs have been usedin patients that do not benefit fromanti-TNF drugs, obtaining good results in terms of remission and long-term prognosis [4]. In the light of the possibility to choose among different biological drugs, with specific targets, the establishment of a personalized treatment is a critical issue. Several efforts have been performed in order to identify biomarkers able to predict the response to treatment and guide the physician in the therapy decision-making. The most promising candidate is TNF, targeted by the mAb infliximab.

\section{${ }^{99 M}$ TC Radiolabelled Infliximab Scintigraphy}

The chimeric mouse/human antibodyinfliximab, demonstrated a greatefficacy in the treatment of patients with inflammatory arthropathies due to its ability to bind TNF with high affinity and specificity both in soluble and membrane form. As demonstrated by recent studies, ${ }^{99 \mathrm{~m}} \mathrm{Tc}$-infliximab scintigraphy could be used to evaluate the presence of TNF at the articular level, in order to evaluate the possibility to treat patients with drugs specifically directed against this molecule [7]. The mAb was radiolabeled bya direct method after reduction of disulphide bond with 2-mercaptoethanol. This allows the binding of ${ }^{99 \mathrm{~m}}$ Tcusing methylen-diphosphonate as a weak competitor. The use of such radiopharmaceutical was reported in the study of Conti et al. in a patient affected by SpA with a mono-arthritis resistant to conventional intra-articular and systemic treatment [7]. After i.v. injection of $370-550 \mathrm{MBq}(100-150 \mu \mathrm{g})$ of ${ }^{99 \mathrm{~m}} \mathrm{Tc}$-infliximab, planar images of the involved joints were acquiredat $6 \mathrm{~h}$ and $24 \mathrm{~h}$.

${ }^{*}$ Corresponding Author: Dr. Fabrizio Conti, Rheumatology, Dipartimento d Medicina Interna e Specialità Mediche, Sapienza Università di Roma, Viale del Policlinico 155, 00161, Roma, Italy; E-mail: fabrizio.conti@uniroma1.it

Citation: Ceccarelli F, Perricone C, Galli F, Valesini G, Conti F (2015) Use of 99mTc-labelled anti-TNF Monoclonal Antibodies to Assess Patients Affected by Inflammatory Arthropathies. Int J Radiol Med Imag 1: 102. doi: http://dx.doi. org/10.15344/ijrmi/2015/102

Copyright: (C) 2015 Ceccarelli et al. This is an open-access article distributed under the terms of the Creative Commons Attribution License, which permits unrestricted use, distribution, and reproduction in any medium, provided the original author and source are credited. 
Citation: Ceccarelli F, Perricone C, Galli F, Valesini G, Conti F (2015) Use of ${ }^{99 m}$ Tc-labelled anti-TNF Monoclonal Antibodies to Assess Patients Affected by Inflammatory Arthropathies. Int J Radiol Med Imag 1: 102. doi: http://dx.doi.org/10.15344/ijrmi/2015/102

Page 2 of 3

Radiolabelled infliximab scintigraphy was useful to assess a high presence of TNF in the right knee justifying the use of anti-TNF drugs. Indeed, the patient was treated by intra-articular infliximab $(100 \mathrm{mg})$ and underwent a control examination at the end of the treatment showing the disappearance of TNF from the inflamed joint. Moreover, the clinical and ultrasonography assessmentconfirmed a total remission. After this case report, a larger population was evaluated including 12 patients with active refractory mono-arthritis (11 knees and 1ankle), affected by RA (5 patients) and SpA ( 7 patients) (8). At baseline and after 12 weeks of treatment with intra-articular infliximab (100 mg for the knee and $50 \mathrm{mg}$ for the ankle), a $99 \mathrm{mTc}$ infliximabscintigraphy was performed. Quantitative analysis of TNF in the inflamed sites was performed using the OSIRIS software. Target to background $(\mathrm{T} / \mathrm{B})$ ratios were calculated drawing circular region of interests (ROIs) on the affected joints (target) andrectangular ROIs approximately five $\mathrm{cm}$ below the knee or above the ankle [8]. The same ROIs wereused to analyse both pre- andpost-therapy images. In order to obtain the T/B ratio for each joint,the activity in the ROIs was normalized and divided by the background activity. The T/B ratio was calculated for affected and non-affected joints onimages acquired at $6 \mathrm{~h}$ and at $20 \mathrm{~h}$. Authors reportedan increasein the T/B ratiofrom 6 to 20 hours. By using this method, the $99 \mathrm{mTc}$-infliximab binding to TNF in the joints was expressed in absolute values and as the percentage increase at $20 \mathrm{~h}$ with respect to $6 \mathrm{~h}(\% \mathrm{~T} / \mathrm{B} 20 \mathrm{~h} / 6 \mathrm{~h})$. The results of these studies confirmed the promising role of ${ }^{99 \mathrm{~m}} \mathrm{Tc}$ infliximab scintigraphy in monitoring the response to anti-TNF therapies. Indeed, a significant decrease in the $\mathrm{T} / \mathrm{B}$ ratio of the affected jointswas associated with complete remission as a confirmation of the success of the treatment. Overall, responder patients showed a significantly greater increase in uptake from $6 \mathrm{~h}$ to $20 \mathrm{hp}$.i. compared to non-responders. Therefore, the high level of TNF confirmed by ${ }^{99 m}$ Tc-infliximab scintigraphy in the inflamed jointsis associated with better efficacy of intra-articular administration of infliximab.

\section{${ }^{99 \mathrm{M}}$ TC Radiolabelled Adalimumab Scintigraphy}

Adalimumab is the first 'fully human' $\mathrm{mAb}$ against TNF approved for the management of moderate to severe active RA and psoriatic arthritis, showingless potential sideeffects and antigenicity compared with infliximab. The administration of this drug seems to be able to down regulate the expression of other pro-inflammatory cytokines, such as IL-6, IL-8 and granulocyte-macrophage colony-stimulating factor [9].

Adalimumabcould be radiolabelled with ${ }^{99 \mathrm{~m}} \mathrm{Tc}$ directly or via an indirect method, using succinimidyl-hydrazinonicotinamide (S-HYNIC) as a bi-functional chelator, tricine as a co-ligand and stannous chloride as a reducing agent. Data from the literature described the comparison between 99mTc-adalimumab and radiolabelled infliximab.

The study conducted by Barrera et al. aimed at assessing the sensitivity and bio-distribution after i.v. administrationof the radiopharmaceutical for TNFin 10 RA patients [2]. Each patient performed two scintigraphic examinationsby the administration of $740 \mathrm{MBq}(0.1 \mathrm{mg})$; whole- body and joint-specific images were acquired after $5 \mathrm{~min}, 4 \mathrm{~h}$, and $24 \mathrm{~h}$.

Results demonstrated that inflamed joints were clearly visualised at 4 and $24 \mathrm{~h}$ p.i. with a median increased uptake of $30 \%$ at $24 \mathrm{~h}$. The uptake was not evident in smaller inflamed joints, suggesting the absence of TNF in some joints. As expected, normal joints did not show uptake in any patients. Patients were then divided into two groups the first one received a therapeutic dose $(10 \mathrm{mg} / \mathrm{kg})$ of unlabelled anti-TNF- $\alpha$ mAb immediately before the ${ }^{99 m}$ Tc-anti-TNF- $\alpha$ mAb administration for competition studies. The second onereceived an intramuscular injection of corticosteroid $(120 \mathrm{mg}) 2$ days before the second scintigraphy to check the sensitivity to reflect decreased inflammation. Results from the first group revealed thatco-injection of unlabelled anti-TNFmAb was able to reduce the uptake of ${ }^{99 \mathrm{~m} T c}$ adalimumab by a median of $25 \%$ as a percentage of injected doses after $24 \mathrm{~h}$. In this way, it was possible to confirm the specificity of the radiopharmaceutical for its target in inflamedjoints. In the second group, systemic corticosteroid administration proved to reduce the disease activity, decreasing uptake of the radiopharmaceutical. This study demonstrated the possibility to use this radiopharmaceutical to detect clinically relevant changes in disease activity [2]. In 2008 Malviya et al. performed a study for therapy decision-making and follow-up with both ${ }^{99 \mathrm{~m}} \mathrm{Tc}$-labelled infliximab and adalimumabmAbs in RA patients [10]. Two groups of patients under went scintigraphic examination with ${ }^{99 \mathrm{~m}}$ Tcinfliximab (12 patients) and ${ }^{99 \mathrm{~m}} \mathrm{Tc}$-adalimumab (9 patients), respectively. Imaging evaluation was performed before and 3 months after intra-articular therapy with infliximab or systemic therapy with adalimumab. After injection of $370 \mathrm{MBq}{ }^{99 \mathrm{~m} T c}-$ infliximab or ${ }^{99 \mathrm{~m}} \mathrm{Tc}$-adalimumab, planar anterior and posterior images of inflamedjoints were acquired at 6 and $20 \mathrm{~h}$ p.i. and T/B ratio was calculated in all affected joints. The authors did not identify differences in the bio-distribution between the two radiopharmaceuticals. A variable degree of joint uptake (T/B ratio ranging from 1 to 4 ) has been observed, which was not always correlated with joint pain or swelling. After the therapy with unlabelled anti-TNFmAb, a reduction in the joint uptake of ${ }^{99 \mathrm{~m}} \mathrm{Tc}$-anti-TNF $\mathrm{mAb}$ correlated with the reduction of clinical symptoms. Moreover, patients withhigher uptake prior to therapy had more benefitthan patients with lower uptake in the inflamed joints. The study conducted by Barrera et al., performing a ${ }^{99 m} \mathrm{Tc}$-adalimumabscintigraphy in patients with active RA before and after treatment with unlabelled anti-TNFmAb or corticosteroids, obtained similar results [2].

\section{Conclusion}

${ }^{99 \mathrm{~m}}$ Tc-labelled anti-TNF mAb scintigraphy is a promising imaging tool that could help to manage and follow-up patients affected by inflammatory arthropathies. ${ }^{99 \mathrm{~m}} \mathrm{Tc}$-infliximab and ${ }^{99 \mathrm{~m}} \mathrm{Tc}$-adalimumab could be candidate for therapy decision-making purposes, predicting the response to anti-TNFtreatment in RA patients.

\section{Competing Interests}

The authors declare that they have no competing interests.

\section{Author Contributions}

All the authors substantially contributed to the literature review, drafting the manuscript and approve the final version of the manuscript.

\section{References}

1. Chianelli M, D'Alessandria C, Conti F, Priori R, Valesini G, et al. (2006) New radiopharmaceuticals for imaging rheumatoid arthritis. Q J Nucl Med Mol Imaging 50: 217-225.

2. Barrera P, Oyen WJ, Boerman OC, van Riel PL (2003) Scintigraphic detection of tumour necrosis factor in patients with rheumatoid arthritis. Ann Rheum Dis 62: 825-828.

3. D'Alessandria C, Malviya G, Viscido A, Aratari A, Maccioni Fet al. (2007) Use of a ${ }^{99 m}$-Technetium labelled anti-TNFa monoclonal antibody in Crohn's Disease: in vitro and in vivo studies.Q J Nucl Med Mol Imaging 51:1-9. 
Citation: Ceccarelli F, Perricone C, Galli F, Valesini G, Conti F (2015) Use of ${ }^{99 \mathrm{~m} T c-l a b e l l e d ~ a n t i-T N F ~ M o n o c l o n a l ~ A n t i b o d i e s ~ t o ~ A s s e s s ~ P a t i e n t s ~ A f f e c t e d ~ b y ~}$ Inflammatory Arthropathies. Int J Radiol Med Imag 1: 102. doi: http://dx.doi.org/10.15344/ijrmi/2015/102

4. Conti F, Ceccarelli F, Massaro L, Cipriano E, Di Franco M, et al. (2013) Biological therapies in rheumatic diseases. Clin Ter 164: e413-428.

5. Sedger LM, McDermott MF (2014) TNF and TNF-receptors: From mediators of cell death and inflammation to therapeutic giants - past, present and future. Cytokine Growth Factor Rev 25: 453-472.

6. Valesini G, lannuccelli C, Marocchi E, Pascoli L, Scalzi V, et al. (2007) Biological and clinical effects of anti-TNFalpha treatment. Autoimmun Rev 7: 35-41.

7. Conti F, Priori R, Chimenti MS, Coari G, Annovazzi A, et al. (2005) Successful treatment with intraarticular infliximab for resistant knee monarthritis in a patient with spondylarthropathy: a role for scintigraphy with ${ }^{99 m} \mathrm{TC}$-infliximab. Arthritis Rheum 52: 1224-1226.

8. Conti F, Malviya G, Ceccarelli F, Priori R, lagnocco A, et al. (2012) Role of scintigraphy with ${ }^{99 m} \mathrm{Tc}$-infliximab in predicting the response of intraarticula infliximab treatment in patients with refractory monoarthritis. Eur J Nucl Med Mol Imaging 39: 1339-1347.

9. Adalimumab (Humira). European Public Assessment Report Scientific Discussion.

10. Malviya G, D'Alessandria C, Lanzolla T, Lenza A, Conti F, Valesini G, et al. (2008) ${ }^{99 m}$ Technetium labelled anti-TNF- $\alpha$ antibodies for the therapy decision-making and follow-up of patients with rheumatoid arthritis. Q J Nucl Med Mol Imaging 52(Suppl 1(2)):13-14. 